\title{
Comparison of two office tests for determining blood theophylline levels
}

\author{
MARK GARRISON, DO \\ JOHN A. BROSE, DO \\ MICHELLE M. RUSSELL, BS \\ JAMES FOGLESONG, DO
}

\begin{abstract}
The blood theophylline level determination is an important therapeutic tool, but reference laboratories can take from 1 to 3 days before giving results. Several quicker in-office methods for testing blood theophylline levels are currently available. Two methods were compared for reliability and accuracy. Both proved to be accurate, but the Acculevel method was more reliable than the Seralyzer method. The Acculevel appears to be a preferable method for in-office testing of serum theophylline levels.
\end{abstract}

The use of theophylline for achieving bronchodilation in chronic obstructive pulmonary disease (COPD) and asthma has been well established by numerous clinical trials. ${ }^{1-3}$ It is commonly accepted that the therapeutic range of theophylline is between 10 and $20 \mu \mathrm{g} / \mathrm{mL} .4,5$ Above this range, potentially serious side effects, such as headaches, agitation, vomiting ${ }^{5}$ and, at worst, sudden death or convulsions, ${ }^{3}$ may occur. Therefore, the family physician must know the patient's blood theophylline level to achieve maximal benefit without harming the patient.

The usual method of theophylline determination is the laboratory standard, high-pressure liquid chromatography (HPLC), ${ }^{6}$ but it usually takes 2 to 3 days to obtain these results. For the family physician in an office setting this delay is not practical, as it would mean a return office visit for the patient. Quicker methods of theophylline determination adapted mostly for office use have been devel- oped over the past 5 years. ${ }^{7,8}$ This study will focus specifically on two in-office methods: Seralyzer (Ames Division, Miles Laboratories, Ltd), and Acculevel (Syntex Laboratories, Inc.) and compare them to the accepted laboratory standard for accuracy. They will then be compared with each other for reliability. The practicality of use for each method in an office setting will be considered.

\section{Methods and materials}

Fifty patients currently taking theophylline preparations and making routine office visits to the Ohio University Osteopathic Medical Center participated in this study. Nurses were instructed to ask all patients taking theophylline if they would participate in this study. Requests were made by telephone to other patients known to be on a theophylline regimen. There were no other inclusion or exclusion criteria.

A 10-mL sample of blood was obtained from each consenting patient. This sample was divided into three equal aliquots. Aliquot $\mathrm{A}$ was centrifuged at 7000 rpm for 5 minutes, after which the serum was stored at $40^{\circ} \mathrm{C}$ until it was transported in a batch to the Ohio State University laboratory for theophylline determination via the HPLC method. Aliquot B was also centrifuged at $7000 \mathrm{rpm}$ for $5 \mathrm{~min}$ utes and the serum kept and divided into two equal parts. Part 1 was assayed immediately by the Seralyzer machine (model 22991), and part 2 was stored at $40^{\circ} \mathrm{C}$.

The Seralyzer machine uses a monoclonal antibody-impregnated reagent strip containing a prepared theophylline enzyme cofactor preparation. The serum sample $(30 \mu \mathrm{L})$ is mixed with distilled 
water $(800 \mu \mathrm{L})$. This dilute sample is placed on a reagent strip and put into the photometer stage of the Seralyzer machine set at a wavelength of $750 \mathrm{~nm}$. Approximately 80 seconds later, the Seralyzer reads out a value. This value is based on reflective photometry produced from the color change induced by the liberated theophylline conjugated to the apoenzyme.

Aliquot $\mathrm{C}$ was not centrifuged, but used as whole blood. This aliquot also was divided into two equal parts: Part 1 was assayed immediately by the Acculevel method, and part 2 was assayed within 60 minutes to check reliability. The whole blood sample cannot be stored for a longer period. The Acculevel method requires no instrumentation; it utilizes enzymes on a strip of chromatographic paper. A $12.5-\mu \mathrm{L}$ sample of the whole blood sample is placed in one packet of reagent solution, and the chromatographic paper is placed in this solution. Capillary action and a subsequent color change while the chromatographic paper is in the developer are then compared with a standard color chart (included in the test packet) to determine the theophylline level. This reaction takes 15 to $20 \mathrm{~min}$ utes.

The other half of aliquot B was stored at $4^{\circ} \mathrm{C}$ and assayed 7 days later by using the Seralyzer to check reliability.

The halves of aliquots $\mathrm{B}$ and $\mathrm{C}$ that were stored at $40^{\circ} \mathrm{C}$ were tested 4 days later by their respective methods to assess repeat reliability. The values obtained by the Seralyzer and Acculevel methods were then compared with the HPLC value to determine the accuracy of these methods against the accepted standard.

\section{Results}

First, the reliability of the Seralyzer method was compared with that of the Acculevel method. We calculated the test-retest correlation coefficients for each machine (0.979 and 0.916 for Acculevel and Seralyzer, respectively). We then used the formula suggested by Snedecor and Cochran ${ }^{9}$ to compare the difference between the two. The Acculevel method was significantly more reliable than the Seralyzer method. $\chi^{2}=12.9, P<.01$ ).

We then looked at the accuracy of both the Seralyzer and Acculevel methods by comparing them with the laboratory standard (HPLC). We first calculated the means of each of the two methods being tested for each subject, and then subtracted each of these means from the HPLC value. In this way, the smaller the number is, the more accurate the method. The dependent $t$ test showed that there was no statistically significant difference in accuracy between the Acculevel (mean $=1.23$ ) method and Seralyzer $($ mean $=1.21)$ methods when compared with HPLC $(P<.94)$.

\section{Discussion}

This study demonstrated that both the Seralyzer and Acculevel methods of theophylline determination are accurate when compared with the recognized laboratory standard (HPLC). In addition, this study showed that the Acculevel method was more reliable than the Seralyzer method; that is, the Acculevel method gave accurate readouts more consistently than the Seralyzer method.

Other aspects to consider when choosing between methods of in-office theophylline testing are cost, convenience, and time expenditure. The Acculevel method appeared to be more cost-effective when only theophylline testing is required and small numbers of samples are being tested. The Seralyzer method requires a machine with a theophyllinedetermining component, reagent strips, and high, low, and control vials. Because the Seralyzer machine must be calibrated every 2 weeks and a control must be run daily, the number of reagent strips necessary is increased. The Acculevel method does not use a machine and therefore requires no calibration or controls; the only expense incurred is for the test packets. For this study, the Seralyzer method cost \$582 more than the Acculevel method.

In terms of convenience and time expenditure, the Acculevel method appeared to be more practical in the office setting. The biweekly calibration and daily control run with the Seralyzer machine may be inconvenient. The Acculevel method does not require instrumentation. Although a control sample of a known level included with each kit of test packets should be run each day blood theophylline determinations are made, this procedure is not essential if one is certain of one's technique and the status of reagents. The time required is slightly longer for the Acculevel than for the Seralyzer method (15-20 minutes vs 80 seconds). But when the length of time from start to finish with the Seralyzer method (including calibration, centrifugation, and the sample) is considered, the time is almost equivalent to that of the Acculevel method. This time equality holds true only if one sample is being tested. When testing multiple samples, the Seralyzer machine is considerably faster.

When reliability, simplicity, and cost were considered in this study, the Acculevel test appeared 


\section{ANEW \\ ANTIHYPERTENSIVE AGENT THAT PROVIDES THERAPEUTIC BENEFITS}
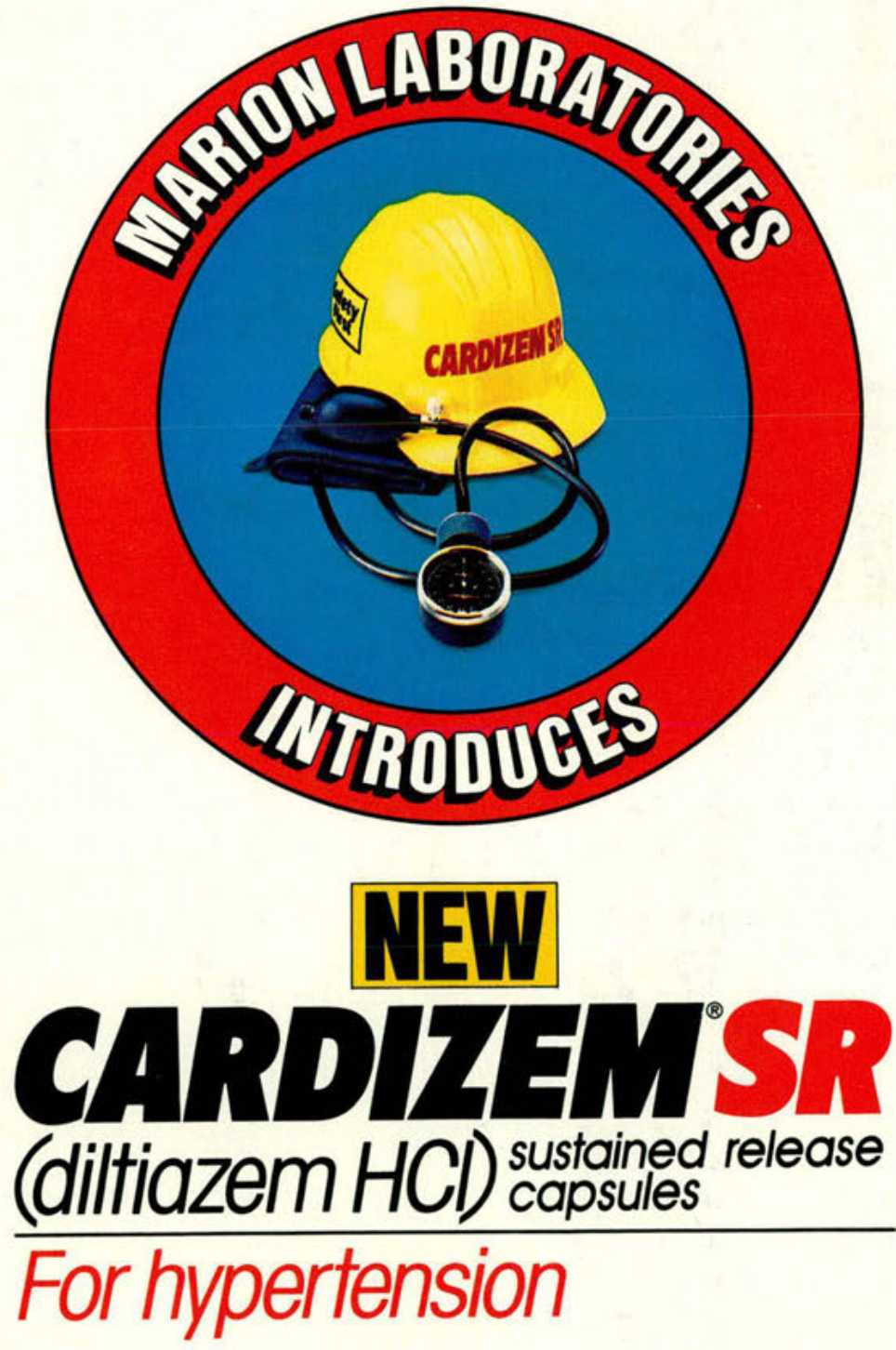


\section{EFFECTIVE THERAPY PLUS HIGH PATIENT AGCEPTANGE}

\section{Effective monotherapy'}

Cardizem SR as monotherapy shows significant blood pressure reduction'

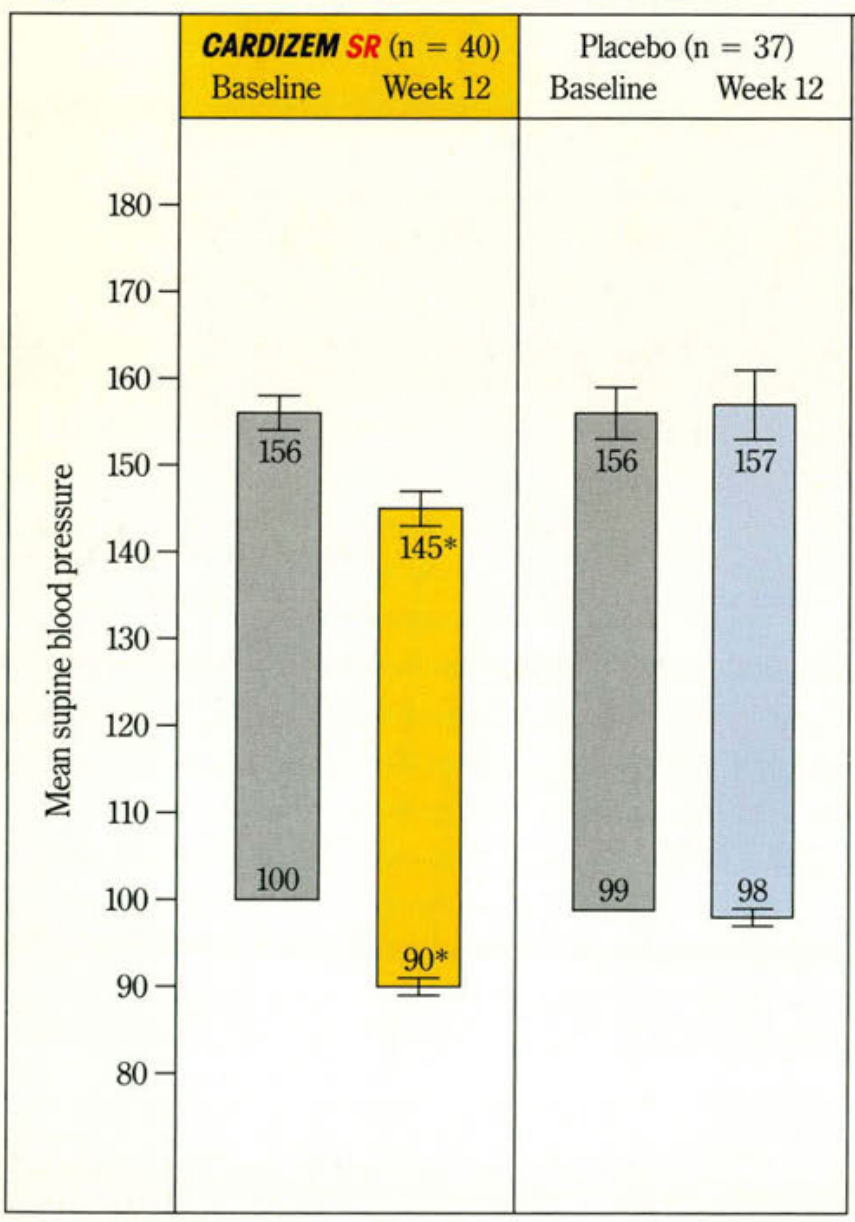

Double-blind study of 77 patients $(14 \%$ black)

Mean age: 57

Dosage: Cardizem SR: up to $360 \mathrm{mg} /$ day

${ }^{*} P<0.01 v$ baseline
High

patient

acceptance

Established safety ${ }^{1}$

Low incidence of CNS side effects, sexual dysfunction, constipation, and other adverse effects $^{1,2}$

Most commonly reported side effects in clinical trials include edema, headache, dizziness, asthenia, sinus bradycardia, flushing and $1^{\circ} \mathrm{AV}$ block

No electrolyte disturbances ${ }^{2,3}$ 


\section{Starting Dosage:}

\section{6 \\ $90 \mathrm{mg}$ bid* \\ Also Available: 120-mg capsules}

"Dosage must be adjusted to each patient's needs, starting with 60 to 120 mg twice daily.

\section{BRIEF SUMMARI}

CARDIZEM SR

(diltiazem hydrochloride)

Sustained Release Capsules

CONTRAINDICATIONS

CARDIZEM is contraindicated in (1) patients with sick sinus syndrome except in the presence of a functioning ventricular pacemaker, (2) patients with secondor third-degree AV block except in the presence of a functioning ventricular pacemaker, (3) patients with hypotension (less than $90 \mathrm{~mm} \mathrm{H} g$ systolic), (4) patients who have demonstrated hypersensitivity to the drug, and (5) patients with acute myocardial infarction and pulmonary congestion documented by $x$-ray on admission.

WARMINGS

1. Cardiac Conduction. CARDIZEM prolongs AV node refractory periods without significantly prolonging sinus node recovery time, except in patients with sick sinus syndrome. This effect may rarely result in abnormally slow heart rates (particularly in patients with sick sinus syndrome) or second- or third-degree AV block (nine of 2.111 patients or $0.43 \%$ ). Concomitant use of dilitiazem with beta-blockers or digitalis may result in additive effects on cardiac conduction. A patient with Prinzmetal's angina developed periods of asystole (2 to

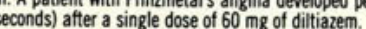

2. Congestive Heart Failure. Although diltiazem has a negative inotropic effect in isolated animal tissue preparations, hemodynamic studies in humans with normal ventricular function have not shown a reduction in cardiac index nor consistent negative effects on contractility (dp/dt). An acute study of oral $24 \%+6 \%$ ) showed improvement in indices of ventricular function without significant decrease in contractile function (do/dt). Experience with the use of SAgnificant decrease in contractile function (dp/dt). Experience with the use of patients with impaired ventricular function is limited. Caution should be patients with impaired ventricular function

3. Hypotension. Decreases in blood pressure associated with CARDIzEM therapy may occasionally result in binod pressure associated

4. Acute Hepatic Injury. Mild elevations of transaminases with and without concomitant elevation in alkaline phosphatase and bilirubin have been concomitant elevation in alkaline phosphatase and bilirubin have been
observed in clinical studies. Such elevations were usually transient and frequently resolved even with continued diltiazem treatment. In rare in stances, significant elevations in enzymes such as alkaline phosphatase, stances, significant elevations in enzymes such as alkaline phosphatase,
LDH, SGOT, SGPT, and other phenomena consistent with acute hepatic iniur have been noted. These reactions tended to occur early after therapy initiation (1 to 8 weeks) and have been reversible upon discontinuation of drug therapy. The relationship to CAROIZEM is uncertain in some cases, but probable in The relationship to CARDIZ
some. (See PRECAUTIONS)

PRECAUTIONS

General. CARDIZEM (diltiazem hydrochloride) is extensively metabolized by the liver and excreted by the kidneys and in bile. As with any drug given over prolonged periods, laboratory parameters should be monitored at regular intervals. The drug should be used with caution in patients with impaired renal or hepatic function. In subacute and chronic dog and rat studies designed to produce toxicity, high doses of diltiazem were associated with hepatic damage. In special subacute hepatic studies, oral doses of $125 \mathrm{mg} / \mathrm{kg}$ and higher in rats were associated with histological changes in the liver which were reversible when the drug was discontinued. In dogs, doses of $20 \mathrm{mg} / \mathrm{kg}$ were also associated with hepatic changes; however, these changes were reversible with continued dosin Dermatological events (see ADVERSE REACTIONS section) may be transient and may disappear despite continued use of CARDIZEM. However skin eruptions progressing to erythema multiforme and/or exfoliative dermatitis have also been infrequently reported. Should a dermatologic reaction persist, the drug should be discontinued.

Drug Interaction. Due to the potential for additive effects, caution and careful titration are warranted in patients receiving CARDIZEM concomitantly with an agents known to affect cardiac contractility and/or conduction. (See WARNINGS Pharmacologic studies indicate that there may be additive effects in prolonging AV conduction when using beta-blockers or digitalis concomitantly with CARDIZEM. (SEe WARNINGS)

As with all drugs, care should be exercised when treating patients with multiple medications. CARDIZEM undergoes biotransformation by chochrome P.450 mixed function oxidase. Coadministration of CARDIZEM with other agents which follow the same route of biotransformation may result in the competitive inhibition of metabolism. Dosages of similarly metabolized drugs, particularty those of low therapeutic ratio or in patients with renal and/or hepatic impairment,

\section{C) 1 D (diltiazem HCl) $\begin{gathered}\text { sustained release } \\ \text { capules }\end{gathered}$}

For hypertension AN AGENT
THAIPROVIDES
THERAPEUTIC BENEFITS

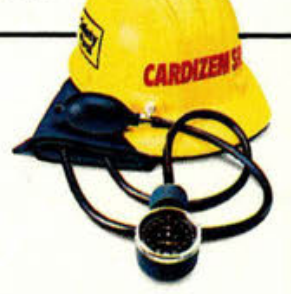

may require adjustment when starting or stopping concomitantly administered CARDIZEM to maintain optimum therapeutic blood levels.

Beta-blockers: Controlled and uncontrolled domestic studies suggest that concomitant use of CARDIZEM and beta-blockers or digitalis is usually well tant treatment in patients with left ventricular dysfunction or cardiac conduction abnormalities.

Administration of CARDIZEM (diltiazem hydrochloride) concomitantly with propranolol in five normal volunteers resulted in increased propranolol levels in all subjects and bioavailability of propranolol was increased approximately $50 \%$. If combination therapy is initiated or withdrawn in conjunction with propranolol, an adjustment in the propranolol dose may be warranted. (See WARNIINGS.)

Cimetidine: A study in six healthy volunteers has shown a significant increase

in peak diltiazem plasma levels (58\%) and area-under-the-curve (53\%) after a in peak diltiazem plasma levels $(58 \%)$ and area-under-the-curve $(53 \%)$ after a 1-week course of cimetidine at $1,200 \mathrm{mg}$ per day and diltiazem $60 \mathrm{mg}$ per day. diated by cimetidine's known inhibition of hepatic cytochrome $P-450$, the enzyme system probably responsible for the first-pass metabolism of diltiazem. Patients currently receiving diltiazem therapy should be carefully monitored for a change in pharmacological effect when initiating and discontinuing therapy with cimetiin pharmacological effect when initiating and discontinuing thera. An adjustment in the diltiazem dose may be warranted

Digitalis: Administration of CARDIZEM with digaxin in 24 healthy male subjects increased plasma digoxin concentrations approximately $20 \%$. Another investigator found no increase in digoxin levels in 12 patients with coronary artery disease. Since there have been conflicting results regarding the effect of digoxin levels, it is recommended that digoxin levels be monitored when initiating, adjusting, and discontinuing CARDIZEM therapy to avoid possible over- or under-digitalization. (See WARNINGS.)

Anesthetics: The depression of cardiac contractility, conductivity, and automaticity as well as the vascular dilation associated with anesthetics may be potentiated by calcium channel blockers. When used concomitantly, anesthetics and calcium blockers should be titrated carefully.

Carcinogenesis, Mutagenesis, Impairment of Fertility. A 24-month study in rats and a 21 -month study in mice showed no evidence of carcinogenicity There was also no mutagenic response in in vitro bacterial tests. No intrinsic effect on fertility was observed in rats.

Pregnancy. Category C. Reproduction studies have been conducted in mice, rats, and rabbits. Administration of doses ranging from five to ten times greater (on a mg/kg basis) than the daily recommended therapeutic dose has resulted in embryo and fetal lethality. These doses, in some studies, have been reported to cause skeletal abnormalities. In the perinatalpostnatal studies, there was some reduction in early individual pup weights and survival rates. There was an increased incidence of stillbirths at doses of 20 times the human dose or greater. There are no well-controlled studies in pregnant women; therefore, use CARDIZEM in pregnant women only if the potential benefit justifies the potential risk to the fetus.

Mursing Mothers. Diltiazem is excreted in human milk. One report suggests that concentrations in breast milk may approximate serum levels. If use of CARDIZEM is deemed essential, an alternative method of infant feeding should

Pediatric Use. Safety and effectiveness in children have not been established. ADVERSE REACTIONS

Serious adverse reactions have been rare in studies carried out to date, but it should be recognized that patients with impaired ventricular function and cardiac conduction abnormalities have usually been excluded from these studies.

The adverse events described below represent events observed in clinical studies of hypertensive patients receiving either CARDIZEM Tablets or CARDIZEM SR Capsules as well as experiences observed in studies of angina and during market. ing. The most common events in hypertension studies are shown in a table with ang. The most common events in hypertension studies are shown in a table with
rates in placebo patients shown for comparison. Less common events are listed by body system; these include any adverse reactions seen in angina studies that were not observed in hypertension studies. In all hypertensive patients studied lover $900)$, the most common adverse events were edema $(9 \%)$, headache $(8 \%)$ diziness $(6 \%)$, asthenia $(5 \%)$, sinus bradycardia (3\%), flushing $(3 \%)$, and $1^{\circ} A V$ block (3\%). Only edema and perhaps bradycardia and diziness were dose related The most common events observed in clinical studies (over 2,100 patients) of angina patients and hypertensive patients receiving CARDIZEM Tablets or CARDIZEM SR Capsules were (ie, greater than $1 \%$ ) edema $(5.4 \%)$, headache $(4.5 \%)$, diziness $(3.4 \%)$, asthenia (2.8\%), first-degree AV block (1.8\%), flushing $(1.7 \%)$, nausea $(1.6 \%)$, bradycardia $(1.5 \%)$, and rash $(1.5 \%)$.

\begin{tabular}{|l|c|c|}
\hline \multicolumn{3}{|c|}{$\begin{array}{c}\text { DOUBLE BLIMD PUCEBO CONTROLLE } \\
\text { HYPERTENSION TRIALS }\end{array}$} \\
\hline Adverse & $\begin{array}{c}\text { Oiltiazem } \\
\text { N=315 } \\
\text { \# pts (\%) }\end{array}$ & $\begin{array}{c}\text { Placebo } \\
\begin{array}{c}\text { N=211 } \\
\text { pts }(\%)\end{array}\end{array}$ \\
\hline headache & $38(12 \%)$ & $17(8 \%)$ \\
\hline AV block first degree & $24(7.6 \%)$ & $4(1.9 \%)$ \\
\hline diziness & $22(7 \%)$ & $6(2.8 \%)$ \\
\hline edema & $19(6 \%)$ & $2(0.9 \%)$ \\
\hline bradycardia & $19(6 \%)$ & $3(1.4 \%)$ \\
\hline ECG abnormality & $13(4.1 \%)$ & $3(1.4 \%)$ \\
\hline asthenia & $10(3.2 \%)$ & $1(0.5 \%)$ \\
\hline constipation & $5(1.6 \%)$ & $2(0.9 \%)$ \\
\hline dyspepsia & $4(1.3 \%)$ & $1(0.5 \%)$ \\
\hline nausea & $4(1.3 \%)$ & $2(0.9 \%)$ \\
\hline palpitations & $4(1.3 \%)$ & $2(0.9 \%)$ \\
\hline polyuria & $4(1.3 \%)$ & $2(0.9 \%)$ \\
\hline somnolence & $4(1.3 \%)$ & - \\
\hline alk phos increase & $3(1 \%)$ & $1(0.5 \%)$ \\
\hline hypotension & $3(1 \%)$ & $1(0.5 \%)$ \\
\hline insomnia & $3(1 \%)$ & $1(0.5 \%)$ \\
\hline rash & $3(1 \%)$ & $1(0.5 \%)$ \\
\hline AV block second degree & $2(0.6 \%)$ & - \\
\hline
\end{tabular}

In addition, the following events were reported infrequently (less than 1\%) have been observed in angina trials. In many cases, the relation to drug uncertain.

Cardiovascular: Angina, armythmia, bundle branch block, tachycardia, ve tricular extrasystoles, congestive heart failure, syncope.

Mervous System: Amnesia, depression, gait abnormality, hallucinations, ne vousness, paresthesia, personality change, tinnitus, trem abnormal dreams.

Gastrointestinal: Anorexia, diarrhea, dysgeusia, mild elevations of SGOT, SGF nd LH (see hepatic warnings), vomiting weight increas thirst.

Dermatological: Petechiae, pruritus, photosensitivity, urticaria

Other: $\quad$ Amblyopia, CPK increase, dyspnea, epistaxis, eve irritatio hyperglycemia, serual difficulties, nasal congestion, nocturis osteoarticular pain, impotence, dry mouth

The following postmarketing events have been reported infrequently in tients receiving CARDIZEM: alopecia, pingival hyperplasia, enthemanty in pe and leukopenia Definitive cause and effect relationship between these even and CARDIZEM therapy cannot yet be established.

Issued $1 / 8$

References: 1. Pool PE, Massie BM, Venkatarama $\mathrm{K}$, et al: $A m$ J Cardiol 1986;57:212-217. 2. Frishmar WH, Kirkendall W, Lunn J, et al: Am J Cardiol 1985 56:92H-96H. 3. Sunderrajan S, Reams G, Bauer JH Hypertension 1986;8:238-242. 4. Frishman WH, Zawad ET Jr, Smith LK, et al: Am J Cardiol 1987;59:615-623.

Another potient benefit product from 1VI PHARMACEUTICAL DIVISION 
to be the preferable method for office theophylline determination when single samples are tested. However, for multiple samples, the Seralyzer machine appears to be more time-efficient.

1. Ellis EF, Koysooko R, Levy G: Pharmacokinetics of theophylline in children with asthma. Pediatrics 1976;58:542-527.

2. Weinberger M: Theophylline for treatment of asthma. $J$ Pediatr 1978:92:1-7.

3. Gilman AG, Goodman LS, Rall TW, et al: Goodman and Gilman's The Pharmacological Basis for Therapeutics, ed 7. New York, Macmillan Publishing Co, 1985 pp 589-603.

4. Hendeles L, Weinberger M, Johnson G: Monitoring serum theophylline levels. Clin Pharmacokinet 1978;3:294-312.

5. Jacobs MH, Senior RM, Kessler G: Clinical experience with theophylline: Relationships between dosage, serum concentration and toxicity. JAMA 1976;235:1983-1986.
6. Adams RF, Vandemark FL, Schmidt GJ: More sensitive high-pressure liquid-chromatographic determination of theophylline in serum. Clin Chem 1976;22:1903-1906.

7. Vaughan LM, Weinberger MM, Milavetz G: Evaluation of the Ames Seralyzer for therapeutic drug monitoring of theophylline. Drug Intell Clin Pharm 1986;20:118-121.

8. Hoffman M, Kiechel F, Meadows CD, et al: Whole blood/plasma ratio determined by a new theophylline test without instrumentation. $J A l$ lergy Clin Immunol 1985;75(suppl):128.

9. Snedecor GW, Cochran WG: Statistical Methods, ed 7. Ames, Iowa, Iowa State University Press, 1980

From the department of family medicine, Ohio University College of Osteopathic Medicine, Athens, Ohio.

Reprints requests to Dr Brose, department of family medicine, Ohio University College of Osteopathic Medicine, Athens, $\mathrm{OH}$ 45701. 